\title{
A String Function for Describing the Propagation of Baroclinic Anomalies in the Ocean
}

\author{
ROBERT H. TYLER* AND ROLF KÄSE \\ Institut für Meereskunde, Abteilung Theoretische Ozeanographie, Kiel, Germany
}

(Manuscript received 14 January 1999, in final form 25 May 2000)

\begin{abstract}
The authors derive a string function that describes the propagation of large-scale, potentially large amplitude, baroclinic energy anomalies in a two-layer ocean with variable topography and rotation parameter. The generality of the two-layer results allows results for the 1-layer, 1.5-layer, inverted 1.5-layer, lens, and dome models to be produced as limiting-cases. The string function is a scalar field that acts as a streamfunction for the propagation velocity. In the linear case the string function is simply $c_{o}^{2} / f$, where $c_{o}$ is the background baroclinic shallow water wave speed, and typically describes propagation poleward on the eastern boundaries, westward (with some topographic steering) over the middle ocean, and equatorward on the western boundaries. In the more general nonlinear case, the string function is locally distorted by the anomaly. In the fully nonlinear examples of a lens or dome, there is no rest or background string function; the string function is generated entirely by the disturbance and propagation is due to asymmetric distribution of the anomalous mass over the string function contours. It is shown that conventional beta/topographic propagation results (e.g., beta drift of eddies, the Nof speed of cold domes) can be obtained as limiting cases of the string function. The string function provides, however, more general propagation velocities that are also usually simpler to derive. The first baroclinic mode string function for the global oceans is calculated from hydrographic data. The westward propagation speeds in the ocean basins as derived from the meridional gradient of the string function are typically two to five times faster than those expected from standard theory and agree well with the propagation speeds observed for long baroclinic Rossby waves in the TOPEX/Poseidon data.
\end{abstract}

\section{Introduction}

An important consideration in large-scale ocean dynamics are the spatial variations in topography and the Coriolis parameter $f$. These cause small convergences in the flow that lead to or alter the propagation of disturbances; examples of this are contained in beta and topographic Rossby waves, the beta drift of large eddies, topographic steering, and the propagation of cold domes along topographic slopes.

In other work (Tyler and Käse 2000a, hereafter TK1) we introduced the "string function" for the case of a homogeneous one-layer model and in a companion paper (Tyler and Käse 2000b), validated these theoretical results using results from a primitive equation model. In this paper we extend that work by considering a twolayer model.

In TK1 the string function for a one-layer model was

* Current affiliation: Applied Physics Laboratory, University of Washington, Seattle, Washington.

Corresponding author address: Dr. Robert H. Tyler, Applied Physics Laboratory, Ocean Physics Department, University of Washington, 1013 NE 40th St., Seattle, WA 98105-6698.

E-mail: tyler@apl.washington.edu easily described as follows: the depth-integrated geostrophic momentum can be decomposed into two parts-a nondivergent part which can be derived from a mass transport streamfunction and a remaining part created by the effects of nonuniform rotation parameter, nonuniform depth, and finite disturbance amplitude. It was shown in TK1 that, regardless of which combination of the latter effects contribute, the remaining divergent momentum component can be described as the product of the mass of the disturbance (due to the dynamic perturbation of the layer thickness) and a propagation velocity. This propagation velocity is nondivergent and can be written in terms of a streamfunction, which we have called the string function. In summary, two points are noteworthy: first, the geostrophic momentum is properly viewed as having a flow part and a propagation part and, second, the velocity of the propagation can be calculated from a scalar string function that encapsulates beta, topographic, and finite amplitude effects simultaneously.

In this paper we derive the string function for the propagation of baroclinic anomalies in a two-layer model. Under the general conditions involving nonuniform topography and rotation parameter and finite amplitudes, the baroclinic and barotropic modes are linearly coupled and therefore are not truly modal. It is, however, useful for the discussion in this paper to describe results 


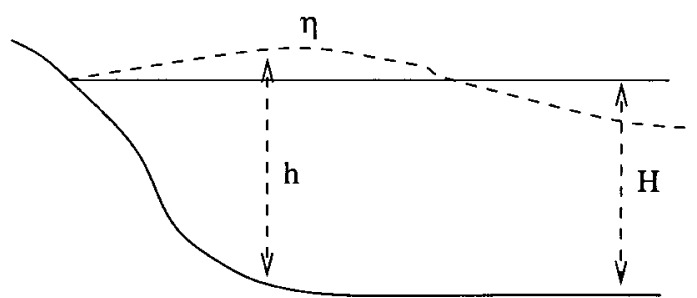

homogeneous 1-layer
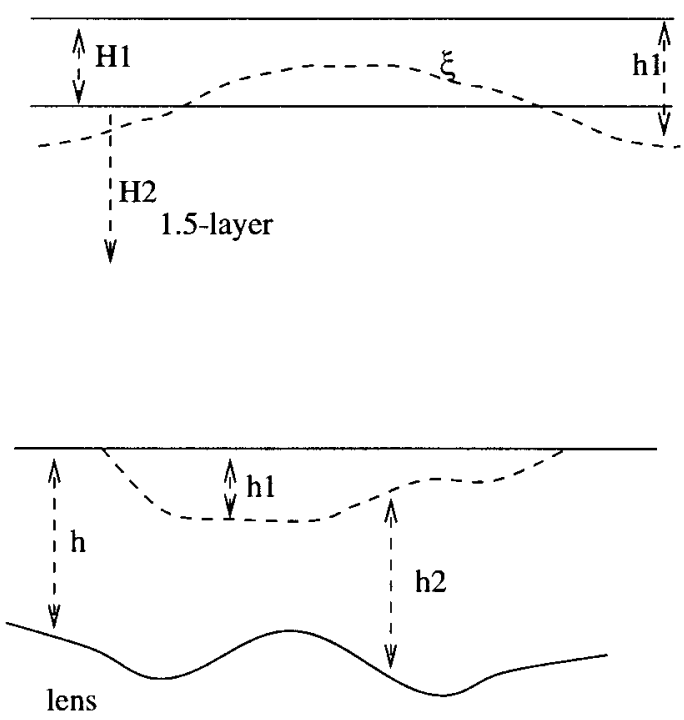
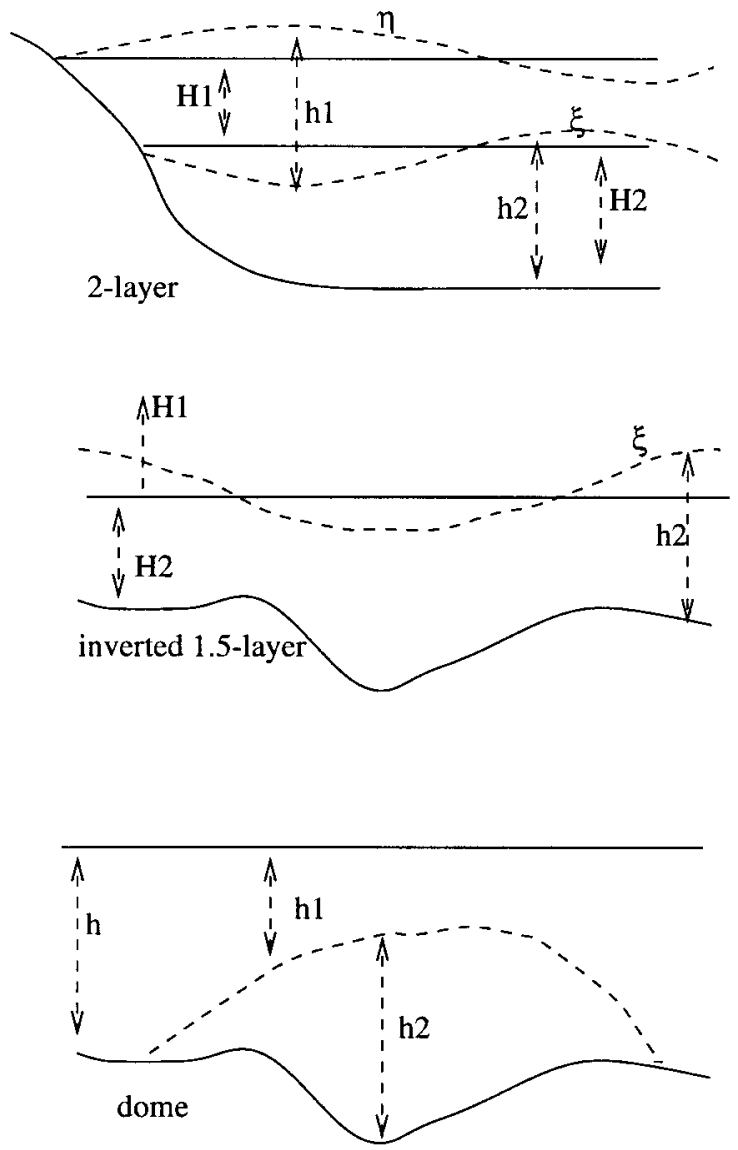

FIG. 1. Schematic of models.

for a separate baroclinic mode and then include consideration of the barotopic coupling terms.

We briefly discuss other previous work, restricting ourselves mostly to those works with which we will later make comparisons. Further references describing related work are given in Tyler and Käse (2000a,b) and the references cited therein.

With exceptions (e.g., Smith and O'Brien 1983; Straub 1994), topographic and beta effects have mostly been examined separately. Earlier work describing the westward propagation of eddies due to the beta effect (e.g., Flierl 1977; McWilliams and Flierl 1979; Nof 1981, 1983a; Killworth 1983; Cushman-Roisin 1986; Shapiro 1986) have been reviewed and a general formulation for the westward drift has been given (Cushman-Roisin et al. 1990). In one of the rarer works dedicated to super-Rossby scales (Matsuura and Yamagata 1982) it is also seen that nonlinearities due to the thickness anomalies become important and can lead to tendencies that counter those of dispersion. In this case, governing equations can take a Korteweg-deVries form for which soliton solutions are possible.

Propagation of eddies and domes along sloped topography have usually been studied while ignoring the beta effect (e.g., Nof 1983b; Swaters 1998; Swaters and Flierl 1991). A simple formula given by Nof (1983b) using a reduced-gravity model has been shown to have some success in predicting the correct order of magnitude of experimentally observed and numerically modeled propagation rates.

In the next section, we present the formulation of the string function for the two-layer model and derive an evolution equation describing the propagation of the potential energy anomaly, or more specifically in this case, the associated mass (thickness) anomaly due to the displaced sea surface and interface. In section 3, we analyze the evolution equation for the case assuming the flow is entirely geostrophic, while in section 4 we include nongeostrophic effects. In section 5 we examine the barotropic coupling term appearing in the baroclinic evolution equation, and in the final three sections we summarize, compare with previous results, and discuss motivation for future development of the string function.

\section{Formulation}

Let us consider the two-layer model shown in Fig. 1. Conservation of mass in each layer implies 


$$
\begin{array}{r}
\partial_{t}\left(\rho_{1} \eta-\rho_{1} \xi\right)+\nabla \cdot \mathbf{s}_{1}=0 \\
\partial_{t}\left(\rho_{2} \xi\right)+\nabla \cdot \mathbf{s}_{2}=0
\end{array}
$$

where $\rho_{1}$ and $\rho_{2}$ are the densities in layer one (upper) and layer two (lower), $\eta$ and $\xi$ are the upward surface and interface displacements, and $\mathbf{s}_{1}$ and $\mathbf{s}_{2}$ are the momentum per unit area for layer one and two (i.e., the integral over the layer depth of the product of density and the horizontal components of velocity).

We will consider dynamics described by the shallowwater, hydrostatic momentum equations in layer integrated flux form. In anticipation of typically geostrophic balances we write these momentum equations in the following form:

$$
\begin{aligned}
& \mathbf{s}_{1}=-\frac{g h_{1}}{f} \boldsymbol{\nabla}\left(\rho_{1} \eta\right) \times \mathbf{z}+\mathbf{R}_{1}, \\
& \mathbf{s}_{2}=-\frac{g h_{2}}{f} \nabla\left(\rho_{1} \eta+\left(\rho_{2}-\rho_{1}\right) \xi\right) \times \mathbf{z}+\mathbf{R}_{2},
\end{aligned}
$$

where $g$ is gravity, $f$ is the Coriolis parameter, $h_{1}$ and $h_{2}$ are the thickness of layer one and two, and $\mathbf{z}$ is the vertical (upward) unit vector. (Familiar forms of the flux-form momentum equations can be obtained by taking the cross product with $\mathbf{z}$ of the forms above and using algebra after expanding $\mathbf{R}_{1}, \mathbf{R}_{2}$ as described next.)

When $\mathbf{R}_{1}=\mathbf{R}_{2}=0$, (3) and (4) describe layer momenta that are in geostrophic balance with the pressuregradient terms. More generally, $\mathbf{R}_{1}$ and $\mathbf{R}_{2}$ describe additional nongeostrophic contributions to the layer momenta. Allowing for nonlinear advection, wind stress ( $\boldsymbol{\tau})$ momentum flux into layer 1, a Rayleigh friction term in layer 2 (with Rayleigh coefficient $b$ which may be nonuniform and has units $s^{-1}$ ), we write

$$
\begin{aligned}
& \mathbf{R}_{1}=f^{-1}\left(\boldsymbol{\tau}-\partial_{t} \mathbf{s}_{1}-\partial_{j}\left(m_{1}^{-1} \mathbf{s}_{1} \mathbf{s}_{1 j}\right)\right) \times \mathbf{z} \\
& \mathbf{R}_{2}=f^{-1}\left(-b \mathbf{s}_{2}-\partial_{t} \mathbf{s}_{2}-\partial_{j}\left(m_{2}^{-1} \mathbf{s}_{2} s_{2 j}\right)\right) \times \mathbf{z},
\end{aligned}
$$

where $m_{1}=\rho_{1} h_{1}$ and $m_{2}=\rho_{2} h_{2}$ are the columnar mass in each layer and summation over repeated indices $(j$ referring to the two horizontal components perpendicular to $\mathbf{z}$ ) is implicit.

Let us now define several functions that will be used in later sections. The first set, which pertains to barotropic dynamics, is

$$
\begin{aligned}
\mathbf{S} & =\mathbf{s}_{1}+\mathbf{s}_{2} \\
\tilde{M} & =\rho_{1} \eta+\left(\rho_{2}-\rho_{1}\right) \xi \\
C & =(g h)^{1 / 2} \\
\Psi_{m} & =\frac{C^{2}}{f} \\
\mathbf{C}_{m} & =\nabla \Psi_{m} \times \mathbf{z},
\end{aligned}
$$

which in sequence are the total momentum $\mathbf{S}$, the total mass anomaly $\tilde{M}$ due to the disturbed layer thicknesses, the barotropic shallow-water wave speed $C$, and the bar- otropic string function $\Psi_{m}$ that acts as a streamfunction for the barotropic generalized beta drift velocity, $\mathbf{C}_{m}$ (or simply "string velocity").

We define a similar set for the baroclinic quantities using lower case:

$$
\begin{aligned}
\tilde{m} & =\rho_{1} \xi \\
c & =\left(\frac{g^{\prime} h_{1} h_{2}}{h}\right)^{1 / 2} \\
g^{\prime} & =\frac{\rho_{2}-\rho_{1}}{\rho_{1}} g \\
\psi_{m} & =\frac{c^{2}}{f} \\
\mathbf{c}_{m} & =\nabla \psi_{m} \times \mathbf{z} .
\end{aligned}
$$

\section{a. Barotropic mode}

Let us add Eqs. (3) and (4) to gain a description of the total momentum S. Using (7)-(12) we write this as

$$
\begin{aligned}
\mathbf{S}= & -\boldsymbol{\nabla}\left(\tilde{M} \Psi_{m}\right) \times \mathbf{z}+\tilde{M} \mathbf{C}_{m}+\mathbf{R}_{1}+\mathbf{R}_{2} \\
& +\boldsymbol{\nabla}\left(\tilde{m} \psi_{m}^{*}\right) \times \mathbf{z}-\tilde{m} \mathbf{c}_{m}^{*},
\end{aligned}
$$

where

$$
\begin{aligned}
& \psi_{m}^{*}=\frac{g^{\prime} h_{1}}{f} \\
& \mathbf{c}_{m}^{*}=\nabla \psi_{m}^{*} \times \mathbf{z} .
\end{aligned}
$$

To interpret Eq. (17) let us first consider the limit of $\rho_{1}=\rho_{2}$. In this case $g^{\prime}=0$ and the starred quantities vanish. The equation then is identical to the one-layer homogeneous case described at length in TK1. Accordingly, (17) states that the total momentum $\mathbf{S}$ is given by a nondivergent component of geostrophic momentum $\left(\tilde{M} \Psi_{m}\right.$ is a mass transport streamfunction for this nondivergent flow), a component of momentum due to the propagation of the total anomalous mass $\tilde{M}$ along the strings with velocity $\mathbf{C}_{m}$, and nongeostrophic components $\mathbf{R}_{1}$ and $\mathbf{R}_{2}$. More generally, when $\rho_{1} \neq \rho_{2}$ the starred stratification-dependent quantities remain.

An evolution equation for $\tilde{M}$ is obtained by using the divergence of (17) together with (8) and the mass conservation equations (1), (2) to yield

$$
\partial_{t} \tilde{M}+\boldsymbol{\nabla} \cdot\left(\tilde{M} \mathbf{C}_{m}-\tilde{m} \mathbf{c}_{m}^{*}\right)+\boldsymbol{\nabla} \cdot\left(\mathbf{R}_{1}+\mathbf{R}_{2}\right)=0 .
$$

Again, in the limit $\rho_{1}=\rho_{2}$ the starred quantity vanishes and (20) is the same as that studied previously in TK1 for a homogeneous one-layer fluid. As brief examples that were described in TK1, when the flow is geostrophic $\mathbf{R}_{1}=\mathbf{R}_{2}=0$ and (20) simply describes generalized beta drift of $\tilde{M}$ with the barotropic string velocity $\mathbf{C}_{m}$, while, when the flow is assumed to be steady and wind stress is retained in the nongeostrophic term, (20) describes steady propagation of $\tilde{M}$ away from the Ekman sources and is a generalized Sverdrup bal- 
ance allowing for nonuniform topography. In the case of uniform topography $\mathbf{C}_{m}$ reduces to the westward long Rossby wave speed $\beta C^{2} / f^{2}$, while in the case of dominant topographic control, $\mathbf{C}_{m}$ produces the long topographic Rossby velocity. More generally, both topographic steering and beta drift enter into $\mathbf{C}_{m}$.

When $\rho_{1} \neq \rho_{2}$ the starred term remains and the equation above, which we will still refer to as describing the barotropic mode, shows coupling with the baroclinic effects.

\section{b. Baroclinic mode}

The layer momentum equations (3) and (4) can be combined into forms that provide more insight into the baroclinic dynamics:

$$
\begin{aligned}
\mathbf{s}_{1}= & \boldsymbol{\nabla}\left(\tilde{m} \psi_{m}\right) \times \mathbf{z}-\tilde{m} \mathbf{c}_{m}+\mathbf{R}_{1}+\frac{h_{1}}{h} \mathbf{S} \\
& -\frac{h_{1}}{h}\left(\mathbf{R}_{1}+\mathbf{R}_{2}\right), \\
\mathbf{s}_{2}= & -\boldsymbol{\nabla}\left(\tilde{m} \psi_{m}\right) \times \mathbf{z}+\tilde{m} \mathbf{c}_{m}+\mathbf{R}_{2}+\frac{h_{2}}{h} \mathbf{S} \\
& -\frac{h_{2}}{h}\left(\mathbf{R}_{1}+\mathbf{R}_{2}\right),
\end{aligned}
$$

where several of the definitions given in (5)-(16) have been used and it is noted that $h=h_{1}+h_{2}$.

Above, (21) describes the momentum in layer one as being the sum of several terms. Taken in order these are 1) nondivergent geostrophic mass transport (given by the streamfunction $\tilde{m} \psi_{m}$ ) due to interface displacement; 2) the momentum due to the generalized beta drift propagating the interface mass anomaly $\tilde{m}$ with velocity $\left.\mathbf{c}_{m} ; 3\right)$ the nongeostrophic components $\mathbf{R}_{1}$ of the momentum in layer 1 ; 4) the last two terms which, taken together, describe the fraction of the geostrophic barotropic momentum that occurs in layer 1. A similar description can be given for (22).

Similarly, we can produce an evolution equation for $\tilde{m}$ by combining (2), (21), and (22) while using (7), (12), and (16) to yield:

$$
\begin{aligned}
\partial_{t} \tilde{m} & +\boldsymbol{\nabla} \cdot\left(\tilde{m} \mathbf{c}_{m}\right) \\
& =-\boldsymbol{\nabla} \cdot\left(\frac{h_{2}}{h} \mathbf{S}\right)-\boldsymbol{\nabla} \cdot\left(\frac{h_{1}}{h} \mathbf{R}_{2}-\frac{h_{2}}{h} \mathbf{R}_{1}\right) .
\end{aligned}
$$

Equation (23) is an evolution equation for the dynamic disturbance of the interface separating two layers. The form of the equation anticipates a situation that is predominantly baroclinic and predominantly geostrophic. In this case the barotropic and nongeostrophic terms appearing on the right are small and the dominant balance is given by the two terms on the left. But the terms on the left simply describe propagation given by the
TABLE 1. Definition of mass anomaly $\tilde{m}$, squared shallow water wave speed $c$, and string function $\psi_{m}=c^{2} / f$ (also separated into background $\psi_{m_{o}}$ and nonlinear $\tilde{\psi}_{m}$ parts) for models shown in Fig. 1 . A general form of $\tilde{m}$ that encompasses all of the models (except the one-layer) is $\tilde{m}=\rho_{1}\left(-h_{1}+H_{1}\right)$ and describes the interfacial mass anomaly with respect to the rest state. Convenient model-specific forms shown can be obtained through sign changes and interchangement of $\rho_{1}$ and $\rho_{2}$ under the Boussinesq approximation.

\begin{tabular}{lccccc}
\hline \hline \multicolumn{1}{c}{ Model } & $\tilde{m}$ & $c^{2}$ & $\psi_{m}$ & $\psi_{m_{o}}$ & $\tilde{\psi}_{m}$ \\
\hline 1-layer & $\rho \eta$ & $g h$ & $\frac{g h}{f}$ & $\frac{g H}{f}$ & $\frac{g \tilde{m}}{f \rho}$ \\
2-layer & $\rho_{2} \xi$ & $\frac{g^{\prime} h_{1} h_{2}}{h}$ & $\frac{g^{\prime} h_{1} h_{2}}{f h}$ & $\frac{g^{\prime} H_{1} H_{2}}{f h}$ & $\frac{g^{\prime} \tilde{m}}{f \rho_{o}}\left(-1+\frac{2 H_{1}}{h}-\frac{\tilde{m}}{\rho_{2} h}\right)$ \\
1.5-layer & $\rho_{2} \xi$ & $g^{\prime} h_{1}$ & $\frac{g^{\prime} h_{1}}{f}$ & $\frac{g^{\prime} H_{1}}{f}$ & $\frac{g \tilde{m}}{f \rho_{1}}$ \\
1.5-lay. (inv.) & $\rho_{2} \xi$ & $g^{\prime} h_{2}$ & $\frac{g^{\prime} h_{2}}{f}$ & $\frac{g^{\prime} H_{2}}{f}$ & $-\frac{g \tilde{m}}{f \rho_{2}}$ \\
Lens & $\rho_{1} h_{1}$ & $\frac{g^{\prime} h_{1} h_{2}}{h}$ & $\frac{g^{\prime} h_{1} h_{2}}{f h}$ & 0 & $\frac{g^{\prime}}{f}\left(\frac{\tilde{m}}{\rho_{1}}-\frac{\tilde{m}^{2}}{\rho_{1}^{2} h}\right)$ \\
Dome & $-\rho_{1} h_{1}$ & $\frac{g^{\prime} h_{1} h_{2}}{h}$ & $\frac{g^{\prime} h_{1} h_{2}}{f h}$ & 0 & $\frac{g^{\prime}}{f}\left(\frac{\tilde{m}}{\rho_{1}}-\frac{\tilde{m}^{2}}{\rho_{1}^{2} h}\right)$
\end{tabular}

baroclinic string function. In the following sections we analyze (23) under varying assumptions.

\section{Case of geostrophic flow}

In this section we examine (23) under the assumption that the flow is in geostrophic balance (i.e., $\mathbf{R}_{1}=\mathbf{R}_{2}$ $=0$ ). In later sections we will add in the nongeostrophic flow effects.

Under this assumption (23) can be written as

$$
\partial_{t} \tilde{m}+\boldsymbol{\nabla} \cdot\left(\tilde{m} \mathbf{c}_{m}\right)=-\boldsymbol{\nabla} \cdot\left(\frac{h_{2}}{h} \mathbf{S}\right),
$$

which, because $\mathbf{c}_{m}$ is nondivergent, can be interpreted as stating that the material derivative of $\tilde{m}$ following the string velocity is zero unless the barotropic term on the right is nonvanishing. The term on the right of (24) simply describes the convergence of that fraction of the total barotropic momentum $\mathbf{S}$ that occurs in layer two. This coupling term is of interest and we will return to it in section 5. First, however, we wish to discuss the terms on the left, in particular we compare them with the one-layer case, and we will assume for expediency that $\mathbf{S}=0$ to start.

In this case (and because $\mathbf{c}_{m}$ is nondivergent) (24) is simply $\partial_{t} \tilde{m}+\mathbf{c}_{m} \cdot \nabla \tilde{m}=0$ describing propagation of $\tilde{m}$ along the strings just as in the one-layer case studied in TK1 except that the definition of the quantities appearing is different in each case. These definitions for the one-layer and two-layer models, as well as other limiting-case models, are summarized in Table 1. Note the similarities in these definitions; $\tilde{m}$ always refers to a mass anomaly due to the alterations in layer thick- 
nesses, while the string function $\psi_{m}=c^{2} / f$ with the shallow water wave speed $c$ chosen appropriately for the model. Also, for a flat-bottomed ocean with vanishing $\tilde{m}$ the string function contours (strings) are aligned with lines of latitude and $\mathbf{c}_{m}=\nabla \psi_{m} \times \mathbf{z}$, calculated using the appropriate string function, correctly reduces to the westward long Rossby speed $\left(\beta c^{2} / f^{2}\right.$ for a beta plane).

Despite these similarities there is an important difference in that the two-layer string function has a different dependence on topography and $\tilde{m}$ than in the onelayer case. Typically, when $h_{1} \ll h_{2}$, the two-layer baroclinic string function will be less sensitive to topography and more sensitive to $\tilde{m}$ than for the one-layer case.

In Table 1 we break the string function into two components: a component $\psi_{m_{o}}$ due to the background configuration (i.e., corresponding to the part of $\psi_{m}$ independent of $\tilde{m}$ ) and the part $\widetilde{\psi}_{m}$ that depends on $\tilde{m}$. Let us first discuss the background string function $\psi_{m_{o}}$ and associated string velocity $\mathbf{c}_{m_{o}}=\nabla \psi_{m_{o}} \times \mathbf{z}$. As described, with uniform topography (and with $H_{1}$ uniform) the background baroclinic string function $\psi_{m_{o}}=g^{\prime} H_{1} H_{2} /$ $(f H)$, where $H=H_{1}+H_{2}$, varies only with $f$ and produces the westward long Rossby wave velocity. In regions where topographic changes dominate $\psi_{m_{o}}$ such as near the coasts, $\mathbf{c}_{m_{o}}=-g^{\prime}\left(H_{1} / H_{2}\right)^{2} f^{-1} \mathbf{z} \times \nabla H$. A zero-value string is located where $\mathrm{H}_{2}$ vanishes and is the boundary for small amplitude $\tilde{m}$ propagation. The general tendency is then for propagation poleward on the eastern boundaries, across the ocean interior and equatorward along the western boundary. This is very much like that for the one-layer model except that the propagation is less sensitive to topography in the open ocean and is typically much slower.

In the 1.5-layer model where we assume an infinite lower layer, $c=\left(g^{\prime} H_{1}\right)^{1 / 2}$ and the string function is therefore $\psi_{m_{o}}=g^{\prime} H_{1} / f$, giving again just the westward long Rossby wave velocity. As can be expected, there is no dependence on topography. Similarly, in the inverted 1.5-layer model (infinite layer on top) $c=$ $\left(g^{\prime} H_{2}\right)^{1 / 2}$ and $\psi_{m_{o}}=g^{\prime} H_{2} / f$, which will typically be very sensitive to topography. In the case where topographic variations dominate, the inverted 1.5-layer string velocity is proportional to the bottom slope.

Now consider the effects of finite amplitude $\tilde{m}$. As seen in Table $1, \tilde{\psi}_{m}$ in the one-layer case is linear in $\tilde{m}$, and therefore the string anomalies have the same sign as $\tilde{m}$. For example, an anticyclonic eddy $(\tilde{m}>0)$ would add a perturbation to the background strings appearing as a bow extending poleward and/or toward shallower water that propagates along the strings; if $\tilde{m}$ is sufficiently large, the bow can even break off, forming a locally closed string. In contrast, the two-layer $\tilde{\psi}_{m}$ has both a term linear in $\tilde{m}$ (similar to the one-layer case but multiplied by a factor $2 H_{1} / H-1$ ) and a quadratic term in $\tilde{m}$, and $\tilde{\psi}_{m}$ need not have the same sign as $\tilde{m}$. Indeed, the sign of two-layer $\tilde{\psi}_{m}$ can change during the propagation as, for example, when a propagating $\tilde{m}$ follows the strings into shallower water.

Consideration of the amplitude of $\tilde{m}$ and $\tilde{\psi}_{m}$ and the changes in amplitude that can occur during propagation is important for determining the degree of nonlinearity in the generalized beta drift; while small amplitude disturbances may follow the background strings in a manner that can be predicted from the outset by examining the background string function, even once small amplitude disturbances can become nonlinear without any external forces, eventually breaking off to form new closed strings that interact with the background strings and perhaps other closed strings. In this case involving breaking and re-attaching strings, the string function still gives the proper propagation velocities, but only in an instantaneous sense because the string configuration is constantly evolving. As described at the end of the last paragraph, sign reversals of $\tilde{\psi}_{m}$ are even possible when strings cross bathymetric contours and enter a region where $1-2 H_{1} / H$ becomes less than zero (i.e., when $H_{2}<H_{1}$ ). But note to see that this can occur, variations in $f$ must be included; otherwise the background strings, at least, would not cross bathymetric contours.

It is also important to note that, in determining the degree of nonlinearity in the generalized beta drift that simple ratios of interface displacement to layer thickness are not satisfactory. Indeed, even the ratio $\bar{\psi}_{m} / \psi_{m_{o}}$, while usually better, is still inadequate. A more reliable indicator is the ratio $\left|\nabla \bar{\psi}_{m}\right| /\left|\nabla \psi_{m_{o}}\right|$ because it estimates the ratio of the disturbance in the string velocity and the background string velocity. For example, while the ratio $\bar{\psi}_{m} / \psi_{m_{o}}$ may increase entering shallow water, suggesting an increase in nonlinearity, increasing topographic slope increases the background string velocity and may predominate to make the disturbance less nonlinear.

We see from Table 1 that the two-layer $\bar{\psi}_{m}$ also has a term quadratic in $\tilde{m}$, which acts to decrease the local value of the string function regardless of the sign of $\tilde{m}$. This will make the asymmetries between the propagation of positive and negative $\tilde{m}$ more complicated. But it is important to note that the terms in $\tilde{m}$ (or $\tilde{m}^{2}$ ) do not complicate the string velocity as much as may appear because the gradients in $\tilde{m}$ do not play a role. This is because any part of $\mathbf{c}_{m}=\nabla \psi_{m} \times \mathbf{z}$, which depends on $\nabla \tilde{m} \times \mathbf{z}$, is perpendicular to $\nabla \tilde{m}$ and therefore cannot advect $\tilde{m}$. This is related to the non-Doppler effect (Rhines 1989).

As can be seen in Table 1, the lens model (1.5-layer model with $H_{1} \rightarrow 0$ ) and dome model (inverted 1.5layer model with $H_{2} \rightarrow 0$ ) have no background string function and any propagation is entirely nonlinear. These models will be discussed in a later section where we make comparisons with previous results. We also note that, in all the models discussed, $\psi_{m}$ is defined such that it is always positive and the $\psi_{m}=0$ contour has the physical significance of describing the location of outcropping. 


\section{Case including nongeostrophic flow}

In this section we relax the restriction of geostrophic flow by allowing $\mathbf{R}_{1}$ and $\mathbf{R}_{2}$ to have nonvanishing values.

To start, we note that the general equation (23) can be rearranged into the form

$$
\partial_{t} \tilde{m}+\boldsymbol{\nabla} \cdot\left(\tilde{m} \mathbf{c}_{m}\right)=-\boldsymbol{\nabla} \cdot\left(\frac{h_{2}}{h} \mathbf{S}_{g}\right)-\boldsymbol{\nabla} \cdot \mathbf{R}_{2},
$$

where $\mathbf{S}_{g}=\mathbf{S}-\mathbf{R}_{1}-\mathbf{R}_{2}$ is the geostrophic component of the barotropic momentum.

Because (25) does not involve $\mathbf{R}_{1}$ explicitly, using (6) it can be cast in a form similar to that for the one-layer case described in detail in TK1. Furthermore, as in TK1, when the momentum is assumed to be predominantly geostrophic, such that momentum appearing in terms on the right can be replaced by geostrophic values and when additionally we assume that the momentum is predominantly baroclinic, we may write:

$$
\begin{aligned}
\partial_{t} \tilde{m}+\boldsymbol{\nabla} \cdot\left(\tilde{m} \mathbf{c}_{m}\right)= & -\boldsymbol{\nabla} \cdot\left(\frac{h_{2}}{h} \mathbf{S}_{g}\right)+\boldsymbol{\nabla} \cdot\left(\frac{b}{f} \psi_{m} \boldsymbol{\nabla} \tilde{m}\right) \\
& -\boldsymbol{\nabla} \cdot \frac{1}{f}\left(\partial_{t} \mathbf{s}_{2}-\partial_{j}\left(m_{2}^{-1} \mathbf{s}_{2} s_{2 j}\right) \times \mathbf{z}\right),
\end{aligned}
$$

which is the same as that studied in TK1 except that the geostrophic barotropic momentum term replaces the Ekman transport term $\boldsymbol{\tau} / \mathbf{f} \times \mathbf{z}$. Hence, the nongeostrophic effects for the two-layer baroclinic case are similar to those in the one-layer case and the criteria derived in TK1 can be applied. For example, for scales larger than the Rossby radius $\left(=\psi_{m} / f\right)$ the dispersion term (involving $\partial_{t} \mathrm{~s}_{2}$ ) and the nonlinear advection term (involving $\partial_{j}$ ) can be neglected relative to $\partial_{t} \tilde{m}$ and the nonlinear string velocity contribution, respectively. In this case (26) simply states that changes of $\tilde{m}$ following the string velocity are due to forcing by convergences of barotropic momentum and dissipation, which appears as diffusion of $\tilde{m}$.

For scales at or below the Rossby radius, the dispersion and nonlinear advection terms on the right can be combined into a term describing material rates of change of layer angular momentum (divided by $f$ ) following the fluid velocity. Neglecting the barotropic and friction terms for the moment and with other assumptions related to neglecting a kinetic energy term (see TK1), the equation is identical in form to that in TK1:

$$
\partial_{t} \tilde{m}+\boldsymbol{\nabla} \cdot\left(\tilde{m} \mathbf{c}_{m}\right)=\partial_{t} \gamma+\boldsymbol{\nabla} \cdot\left(\gamma \mathbf{u}_{2}\right),
$$

where $\gamma=\mathbf{z} \cdot \boldsymbol{\nabla} \times \mathbf{s}_{2} / f$ is proportional to the angular momentum (per $f$ ) and $\mathbf{u}_{2}=\mathrm{s}_{2} / m_{2}$ is the velocity in layer 2. Equation (27) states that changes in $\tilde{m}$ following the string velocity are balanced by changes in $\gamma$ following the velocity. That is, the dynamics are controlled by advection (propagation really) of mass by the string ve- locity, and advection of angular momentum by the flow velocity.

\section{Barotropic coupling}

As we described in the introduction, in the general two-layer case involving finite amplitudes and topography the barotropic and baroclinic modes remain linearly coupled. The two evolution equations, (20) and (23), would need to be solved simultaneously. But given the typically different time and space scales for the two modes it is useful to attempt to make inferences about the effect of the barotropic mode on the baroclinic propagation.

For simplicity let us assume that the space scales considered are larger than the internal Rossby radius, and friction is such that the flow in layer two is approximately geostrophic and therefore $\mathbf{R}_{2}$ can be neglected. The governing equation (25) is then

$$
\partial_{t} \tilde{m}+\boldsymbol{\nabla} \cdot\left(\tilde{m} \mathbf{c}_{m}\right)=-\boldsymbol{\nabla} \cdot\left(\frac{h_{2}}{h} \mathbf{S}_{g}\right) .
$$

Let us first give a simple interpretation of the barotropic effects in (28) in terms of the barotropic velocity. By defining a depth-averaged velocity as $\mathbf{U}=\mathbf{S}_{g} /\left(\rho_{1} h\right)$ and noting $h_{2}=H_{2}+\tilde{m} / \rho_{2}$, (28) can be written as

$$
\partial_{t} \tilde{m}+\boldsymbol{\nabla} \cdot\left[\tilde{m}\left(\mathbf{c}_{m}+\mathbf{U}\right)\right]=\boldsymbol{\nabla} \cdot\left(\rho_{1} H_{2} \mathbf{U}\right),
$$

which shows that the normal propagation along the baroclinic strings is Doppler shifted by $\mathbf{U}$ and a source term on the right remains and can be expected to be important when barotropic flow flows across bathymetric contours.

Although (29) provides insight, $\mathbf{U}$ must be prescribed and the constraints given by the equations governing the barotropic dynamics have not yet been imposed. To improve on this, we use (17) to replace for the geostrophic component of the barotropic momentum $\mathbf{S}_{g}=$ $\mathbf{S}-\mathbf{R}_{1}-\mathbf{R}_{2}$ in (28) to yield

$$
\partial_{t} \tilde{m}+\boldsymbol{\nabla} \cdot\left(2 \tilde{m} \mathbf{c}_{m}\right)=\boldsymbol{\nabla} \cdot\left(\frac{g h_{2}}{f} \boldsymbol{\nabla} \tilde{M} \times \mathbf{z}\right),
$$

where we have used (10), (15), (16), and vector identities.

It is interesting to compare (28) with (30); if we assume $\mathbf{S}_{g}=0$ (no net momentum), then (28) describes simple propagation along the strings at the string velocity. Alternatively, if we assume instead that $\tilde{M}=0$ (no net mass) then by (30) the situation is similar except that the propagation is at twice the speed of the string velocity. Which, if either, of these assumptions is appropriate?

To address this, let us examine the evolution equation for $\tilde{M}$ (20). Specifically, let us consider the steady geostrophic version of this equation. In this case the two string velocity terms must balance. These terms appear as the divergences of the momentum fluxes $\tilde{M} \mathbf{C}_{m}$ and $\tilde{m} \mathbf{c}_{m}^{*}$, and it is hard to make any rigorous claims about 
relative magnitudes of $\tilde{M}$ and $\tilde{m}$ that do not depend on assumptions of a specific configuration. Instead, we consider estimating the range of the parameter $\epsilon$ for an assumed relationship:

$$
\tilde{M}=\epsilon \frac{g^{\prime}}{g} \tilde{m} .
$$

Noting that the string velocities are divergenceless and assuming that we evaluate the mass gradient terms on the same spatial scale, let us require that $\tilde{M} \mathbf{C}_{m}$ and $\tilde{m} \mathbf{c}_{m}^{*}$ balance (this is more restrictive than assuming just that their divergences balance). From this and the definitions of the string velocities, we estimate $\epsilon$ as

$$
\epsilon=\frac{\left|\nabla\left(h_{1} f^{-1}\right)\right|}{\left|\nabla\left(h f^{-1}\right)\right|} .
$$

Inspecting (32), it appears that when topographic variations dominate variations in $f$ and the amplitudes are small such that $h_{1} \approx H_{1}$ (which is uniform), we have $\epsilon$ $\approx 0$. When the $f$ variations dominate (simple beta drift with no topographic influence), we have $\epsilon \approx h_{1} / h \leq 1$. Hence, within the validity of the restrictive assumptions we have made, we expect $\epsilon$ to range from 0 to 1 . Physically, $\epsilon=0$ gives $\tilde{M}=0$ and describes the situation of compensation $\eta=-\left(g^{\prime} / g\right) \xi$, while $\epsilon=1$ describes the situation $|\eta| \ll\left|g^{\prime} / g \xi\right|$ in which little mass anomaly is carried in the sea surface displacement.

Using (17) in (28) together with (31) and $\mathbf{S}_{g}=\mathbf{S}-$ $\mathbf{R}_{1}-\mathbf{R}_{2}$, we can write

$$
\partial_{t} \tilde{m}+\boldsymbol{\nabla} \cdot\left(\tilde{m} \mathbf{c}_{m}^{\#}\right)=0,
$$

where $\mathbf{c}_{m}^{\#}=\nabla \psi_{m}^{\#} \times z$ uses the modified string function

$$
\psi_{m}^{\#}=\left(2-\epsilon \frac{h}{h_{1}}\right) \psi_{m} .
$$

This result predicts that, when the generalized beta drift is controlled by topography, $\epsilon \approx 0$ and the disturbance $\tilde{m}$ propagates with twice the usual string velocity due to the barotropic coupling; when the beta effect is predominant, however, we have $\epsilon \approx 1$ and the propagation is at the usual string velocity (which then corresponds to the westward baroclinic long-Rossby wave speed).

We emphasize that several assumptions have been made in this section that limit the validity of the results presented. For particular applications these should be reviewed.

\section{Summary of theoretical results}

To summarize our theoretical results, the evolution equations for any of the models shown in Fig. 1 can be written as

$$
\partial_{t} \tilde{m}+\boldsymbol{\nabla} \cdot\left(\tilde{m} \mathbf{c}_{m}\right)=-\boldsymbol{\nabla} \cdot\left(\mathbf{S}_{F}+\mathbf{S}_{b}+\mathbf{S}_{I}\right) .
$$

For any model, the string velocity is $\mathbf{c}_{m}=\boldsymbol{\nabla} \psi_{m} \times \mathbf{z}$ and the string function is $\psi_{m}=c^{2} / f$. Hence, it is only through the different definitions (see Table 1) for the mass disturbance $\tilde{m}$ and the shallow water wave speed $c$ that the models differ from one another regarding the generalized beta drift described by the terms on the left of (35). Because the string velocity $\mathbf{c}_{m}$ is nondivergent, the left side of (35) can also be viewed as the total derivative following $\mathbf{c}_{m}: \partial_{t} \tilde{m}+\mathbf{c}_{m} \cdot \boldsymbol{\nabla} \tilde{m}$. Changes in $\tilde{m}$ following the string velocity are due to the convergences in the various momentum fluxes shown on the right. For many cases these right-side terms vanish and the evolution of $\tilde{m}$ is determined entirely by $\mathbf{c}_{m}$. Even in this case, the dynamics need not be simple because of the shear in $\mathbf{c}_{m}$ and because of nonlinear effects entering through the dependence of $\mathbf{c}_{m}$ on $\tilde{m}$ of the string velocities.

The momentum terms on the right of (35), in sequence, are $\mathbf{S}_{F}$, which in the case of the one-layer model is the Ekman momentum $\left(=f^{-1} \boldsymbol{\tau} \times \mathbf{z}\right)$, while in the baroclinic models it is the fraction of the geostrophic barotropic momentum in the lower layer $\left[=\left(h_{2} / h\right) \mathbf{S}_{g}\right]$; $\mathbf{S}_{b}=f^{-1} b \mathbf{S}_{2} \times \mathbf{z}$, which is the momentum flux due to Rayleigh friction in the lower layer (simply use $\mathrm{s}$ for the one-layer model); and finally

$$
\mathbf{S}_{I}=f^{-1}\left[\partial_{t} \mathbf{S}_{2}+\partial_{j}\left(\frac{1}{m_{2}} \mathbf{s}_{2} s_{2 j}\right)\right] \times \mathbf{z},
$$

the divergence of which is related to changes in layer angular momentum as described in the last section (again, simply use $\mathbf{s}$ instead of $\mathbf{s}_{2}$ for the one-layer model). When we assume that $\mathbf{s}_{2}$ is geostrophic to first order, the friction term can be transformed into a Laplacian diffusion term for $\tilde{m}$, and for scales larger than the Rossby radius, $\mathbf{S}_{I}$ becomes negligible.

\section{Comparisons with previous results and discussion}

In the small amplitude limit, it is simple to show in a manner similar to that done in TK1 that the predictions given by the string function are consistent with the theory for linear topographic and beta Rossby waves. Here, we will focus our comparison on isolated nonlinear features such as eddies, lenses, and domes, with possibly large amplitudes. We will first compare with propagation velocities expected from the baroclinic string function and then discuss possible adjustments to this due to coupling with the barotropic mode.

The string function describes the propagation under the combined influences of topography and nonuniform $f$ and, in the large amplitude case, nonlinear effects due to the dependence of the string function on $\tilde{m}$. To compare with previous results we first consider the two regimes (purely topographic or beta drift) separately.

In the case of the westward motion of isolated surface anomalies due to beta drift in a reduced-gravity 1.5layer ocean on a beta plane (and assuming $g^{\prime} \ll g$ ) the comparison can be done quickly by showing that our results in this limit agree with the general equation given 
in Cushman-Roisin et al. (1990), which was shown to encompass many previous results. They obtained (in our notation)

$$
\mathbf{U}_{\mathrm{cm}}=-\frac{\beta g^{\prime}}{f_{o}^{2}} \frac{\int_{A}\left(H_{1} \tilde{m}+\tilde{m}^{2}\left(2 \rho_{o}\right)^{-1}\right) d A}{\int_{A} \tilde{m} d A} \hat{x}
$$

where $f=f_{o}+\beta y, \rho_{o}$ is a reference water density (because $g^{\prime} \ll g \rho_{o} \approx \rho_{1} \approx \rho_{2}$ here), and $\int_{A}$ ( ) $d A$ is the area integral over the anomaly.

It can be shown that the center of mass velocity $\mathbf{U}_{\mathrm{cm}}$ above is simply the mass-weighted string velocity (i.e., total momentum $\int_{A} \tilde{m} \mathrm{c}_{m} d A$ divided by total mass $\int_{A} \tilde{m}$ $d A$ ), where $\mathbf{c}_{m}$ depends on $\tilde{m}$. This result agrees with our results for either the lens or 1.5-layer models when we impose in our results the assumption of predominantly geostrophic flow, assume that the area integral of the gradient of the mass transport streamfunction $\tilde{m} \psi_{m}$ vanishes, and neglect any coupling with the barotropic mode. Interpreting (36) in our context, we can say that the term involving $H_{1}$ in (36) is due to propagation of $\tilde{m}$ by the background strings $\psi_{m_{o}}$, while the $\tilde{m}^{2}$ term is due to the propagation by the nonlinear component of the strings $\tilde{\psi}_{m}$.

Cushman-Roisin et al. also extended the analysis of westward drift to a two-layer flat-bottom case. They found equations describing the drift in each layer that agree with those for our two-layer case in the flat-bottom limit when barotropic effects are discarded. From the crude arguments presented in section 5 when the string velocity is dominated by the variation in $f, \epsilon \approx 1$ and influence of the barotropic mode is expected to be negligible. Similar flat-bottom results typically with stronger restrictions are discussed in Flierl (1984), Nof (1981), and Killworth (1983).

Now let us compare with results for propagation when topography is the dominant factor. First, let us make a distinction between "walls" and topographic slope. In the string function formulation there are no walls. Walls would imply "string ends," which do not exist except perhaps at the equator. There is a near equivalent, however. For example, as strings reach the western side of a basin and turn equatorward, they can become tightly bunched together in regions of strong slope. As $\tilde{m}$ attempts to follow the strings $\tilde{m}$ becomes stretched and the cross-string length scale decreases leading to strengthening of the nongeostrophic components. If the flow remains linear, dispersion is the primary new effect and in the limit of infinite string density near the boundary, the linear evolution equation gains a form that can be interpreted as describing Kelvin wave decay away from the boundary. When the amplitudes are great, the vorticity/angular momentum and kinetic energy must be considered. Such an approach may give results in agreement with previous wall phenomena, such as the image effect described in Käse (Käse and Zenk 1996) or the "wodons" described by Shi and Nof (1994). We will not attempt a comparison with the previous wall results but only consider results for propagation due to topographic slope.

First we will consider the case of a dome where the upper layer is assumed infinite and the $f$ variations are ignored and we will show that the result in this restricted case is the same as that found by Nof (1993b). From Table 1, the appropriate dome string function [with Nof's assumption $h_{2} \ll h_{1}$ (and implicitly, $\partial_{t} h \approx 0$ ) and uniform $f=f_{o}$ ] is $\psi_{m} \approx g^{\prime} h_{2} / f_{o}$ and the dome mass anomaly is $\tilde{m}=-\rho_{2} h_{1}$. Using $h_{2}=h-h_{1}$ the propagating momentum is $\tilde{m} \mathbf{c}_{m}=\tilde{m} \boldsymbol{\nabla}\left(g^{\prime} h / f_{o}\right) \times \mathbf{z}-$ $\boldsymbol{\nabla}\left[g^{\prime} \rho_{1} h_{1}^{2} /(2 f)\right] \times \mathbf{z}$. The last term is nondivergent and can be dismissed; then the propagation velocity is proportional to slope and agrees with Nof's result. Rigorously, we have only proved that $\tilde{m}$ (and therefore $h_{1}$ ) propagates with the Nof speed; Nof found the propagation of $h_{2}$. It can be easily shown that $h_{2}$ in our results also propagates with the string velocity above by expanding $\tilde{m}=-\rho_{1} h+\rho_{1} h_{2}$ and dismissing the nondivergent term $g^{\prime} \rho_{1} h / f \nabla h \times \mathbf{z}=\nabla\left[g^{\prime} \rho_{1} h^{2} /(2 f)\right] \times \mathbf{z}$.

Experimental observations of baroclinic eddies on sloping bottoms (Mory et al. 1987; Whitehead et al. 1990) give conflicting results, particularly regarding the cross-isobath component of propagation. There appears to be order of magnitude verification of the Nof velocity along the isobaths in the case of Whitehead et al. (1990), while the observed translation along the isobaths in the Mory et al. (1987) experiments were typically an order of magnitude less than the Nof prediction. We note that in the latter experiments the upper and lower layers were the same order of magnitude. The dome string velocity calculated without Nof's assumption $h_{2} \ll h_{1}$ predicts speeds smaller than the Nof speed by a factor $\left(h_{1} / h\right)^{2}$ $\approx 1 / 4$, giving results closer in agreement to the Mory et al. observations.

Another factor is that in both of the experiments above the velocities in the upper layer above the dome were large, in disagreement with Nof's assumption regarding a reduced-gravity state. In some cases the experiments suggest that the momentum above the dome exceeds that within the dome. More recently (Swaters and Flierl 1991; Swaters 1998), work has appeared that includes extensions to propagating domes that interact with the surrounding fluid and for which the velocities in the upper layer agree better with the experimental observations. The propagation velocity is still, however, in first-order agreement with that of Nof. The numerical results of Swaters (1998) showed speeds $20 \%$ less than the Nof speed and this difference was attributed to the interaction between the dome and the surrounding topographic Rossby wave field.

Regarding the effect of barotropic coupling, from the crude estimates in section 5 it appeared that for the case of strong topographic control such as for the dome 
$\tilde{M} \approx 0$ (mass compensation) and the effect is to increase the string velocity by a factor of 2 .

Now we will compare our results with previous formulations that have included the effects of a prescribed barotropic flow such as discussed in Section 5. As reviewed in Rhines (1993), a time-dependent equation appropriate to large-scale, slowly varying circulation in a two-layer ocean of uniform total depth $h$ is (with some changes in notation)

$$
\partial_{t} h_{1}+\mathbf{U} \cdot \boldsymbol{\nabla} h_{1}-\frac{\beta g^{\prime} h_{1} h_{2}}{f^{2} h} \partial_{x} h_{1}=-\frac{h_{2}}{h_{1}} \boldsymbol{\nabla} \times \frac{\boldsymbol{\tau}}{f},
$$

which can be easily obtained from (29) assuming a flat bottom, $\boldsymbol{\nabla} \cdot \mathbf{U}=0$, and including wind stress.

Another important comparison can be made with the governing equation given by Rhines (1989) for largescale slowly varying circulation in an inverted (infinite layer on top) 1.5-layer ocean with nonuniform topography. His equation (again with some notation changes) is

$$
\partial_{t} \zeta+a_{1} \partial_{x} \zeta+a_{2} \partial_{y} \zeta=\mathbf{U} \cdot \boldsymbol{\nabla} d+K \nabla^{2} \zeta,
$$

where $a_{1}=U-\left(g^{\prime} / f\right) \partial_{y} d-\beta g^{\prime} h f^{2}, a_{2}=V+$ $\left(g^{\prime} / f\right) \partial_{x} d, f=f_{o}+\beta y,(U, V)$ are the east/west components of $\mathbf{U}$, and $d$ is the topographic height above a reference uniform total depth. Equation (38) can be obtained from (29) when we assume $\boldsymbol{\nabla} \cdot \mathbf{U}=0$, and Rayleigh friction is included (the nongeostrophic Rayleigh friction momentum terms discussed can be converted to a Laplacian diffusion term for $\tilde{m}$ by making the assumption that the flow is predominantly geostrophic).

The Rhines (1989) paper also contains other discussions (e.g., concerning wave characteristics and the nonDoppler effect), which anticipates results from the string function formulation. The wave characteristics obtained by ray tracing are often simply contours aligned with the string function and similarly contain the information about propagation paths when topography and beta are important. As discussed, in the string function context, the non-Doppler effect arises because components of $\mathbf{c}_{m}$ that are purely a function of $\tilde{m}$ cannot have an effect because they are directed perpendicular to the gradients in $\tilde{m}$.

We close this section by drawing attention to a case previously discussed by Shi and Nof (1994) in a similar theoretical context. A Gulf Stream warm-core ring (82B) near the western Atlantic continental slope was observed to propagate southwestward while moving upslope. This propagation did not fit that expected for a wodon (Shi and Nof 1994). Cornillon et al. (1989) argued that such rings move southward because of interactions with the flow, and Flierl (1984) considered it possible that the combination of westward beta drift and radiated Rossby wave drag could be responsible for the observed propagation.

Without discounting these possibilities, we will point out that a rough estimate of the regional string function (using the data discussed in Shi and Nof, or simply the estimates in Fig. 2, which will be discussed) suggests that ring $82 \mathrm{~B}$ propagates as a well-behaved string anomaly. The propagation moves along the $7300 \mathrm{~m}^{2} \mathrm{~s}^{-1}$ string and at a speed of a few centimeters per second, which also agrees with the string velocity. While the strings in this region depend much on topography, the beta effect is also important causing the strings (and propagation of ring $82 \mathrm{~B}$ ) to cross topography and move upslope.

\section{Future work}

There are at least two important motivations for further developing the string function formulation. First, in studies of the propagation of large-scale energy anomalies under combined beta and topographic effects, the string formulation provides more generality than previous approaches-easily incorporating topography and beta simultaneously - and plots of the string function will usually give more insight than plots of other quantities such as potential vorticity or geostrophic contours. In this sense, the string function can be offered as an encompassing yet simpler interpretation of previous formulations.

The string function can also be used to address timely issues that have not yet been resolved. In particular, we suggest that the string formulation can be practically exploited for studying the current disparity between theoretical and observed Rossby wave propagation characteristics.

A recent article by Chelton and Schlax (1996) describes long ocean Rossby waves observed by the TOPEX/Poseidon altimetry satellite. The observed slowly propagating waves are held to be first-mode baroclinic Rossby waves. Several noted features of the waves are that the phase speeds are usually larger than that predicted by standard theory with particular phase speed increases over midocean ridges and in the western parts of the basins, and that the waves often appear to be generated at the midocean ridges.

The increase of phase speeds in the western part of the basin can be expected from the local increase in the upper-layer thickness, and the generation of the waves over topography, while not completely understood, is not surprising because various mechanisms such as barotropic flow across isobaths will charge the baroclinic modes in these regions.

Probably the most peculiar of the observations is the rather ubiquitous departure of the westward phase speeds from theory and the enhancement of the phase speeds over topographic features. Important recent papers (Dewar 1998, Killworth et al. 1997) address this topic; yet further work to settle this issue is required. According to the standard theory for long linear Rossby waves, the westward phase speed in this case should be $\beta c^{2} / f^{2}$, where $c^{2}$ is the first baroclinic mode shallow water wave speed. Using an estimate of the nonuniform $c$ based on hydrographic data (Chelton et al. 1998), 


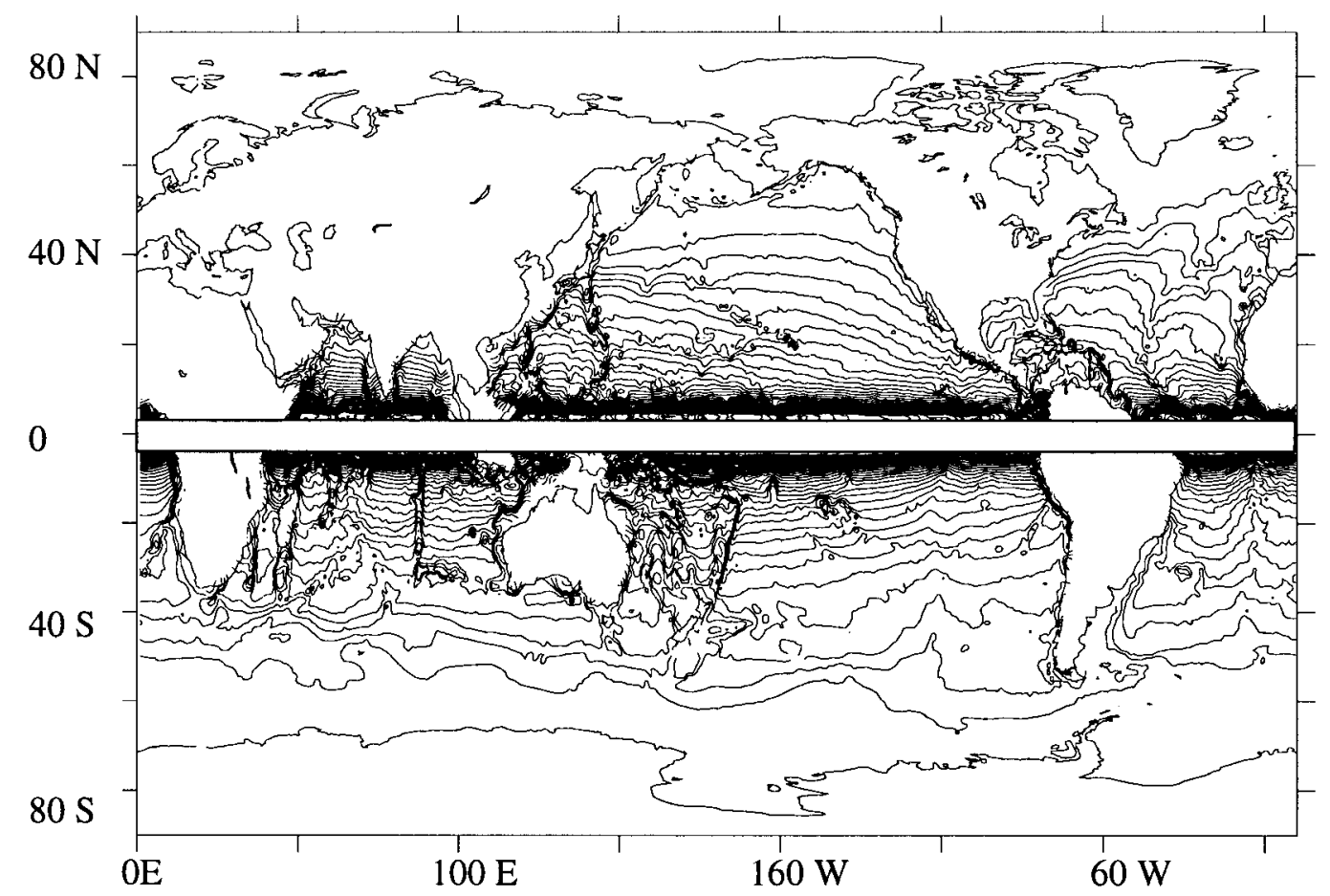

FIG. 2. First baroclinic string function $\psi_{m}=c^{2} / f\left(\mathrm{~m}^{2} \mathrm{~s}^{-1}\right)$ for extratropical globe. [Here $c$ is the first baroclinic shallow water wave speed estimated by Chelton et al. (1998) from hydrographic data]. Contour interval is 20000 $\mathrm{m}^{2} \mathrm{~s}^{-1}$. [Values are 0 at the coasts, the first $\left(=20000 \mathrm{~m}^{2} \mathrm{~s}^{-1}\right)$ contour appears typically as the most poleward contour in each ocean and values increase then equatorward.]

Chelton and Schlax (1996) were able to calculate the globally varying theoretical westward phase speed. From this, and taking zonal averages, they were able to calculate the ratio of the observed phase speeds to the theoretical value. Their findings (clumped together here for all oceans) are shown in Fig. 4. As can be seen, the observed phase speeds are typically a couple to several times greater than that theoretically expected, and the discrepancy shows an increase poleward.

In light of these results, it is useful to attempt to apply the results presented in this paper to the realistic global oceans. Of course the results we have presented apply to a two-layer ocean, not one that is realistically stratified; any comparisons are intended only to motivate further research rather than to explain the observations. There are also other difficulties with such comparisons that will be discussed.

Let us consider the question, "what long Rossby wave propagation velocities do we expect for a twolayer ocean having the same topography as the ocean and the same distribution of baroclinic shallow water wave speed $c$ ?" From $c$ we can easily calculate the baroclinic string function $\psi_{m}=c^{2} / f$ and from the gradient the string velocity $c_{m}$ is obtained.

The string function for the first baroclinic mode is shown in Fig. 2 [calculated using estimates of $c$ provided by D. Chelton and described in Chelton et al. (1998)]. The westward phase speed is calculated from the me- ridional gradient of the string function and the ratio of this speed to that expected from standard linear Rossby wave theory is shown in Fig. 3. As can be seen, the westward speeds expected from the string function are rather different than the description given by the standard theory. In particular, topographic gradients (which do not enter the standard theory) clearly play an important role.

Now we simply take the zonal average of the westward speed ratio shown in Fig. 3 and plot it in Fig. 4 to compare with the satellite observed speeds. As can be seen, both the observed and string speeds are typically several times the theoretical speeds at high latitudes and the ratio increases poleward. When we consider the scatter in the satellite data and the regionality of the westward speed expected from Fig. 3, the correlation between the phase speeds predicted by the string function and those observed is surprisingly good.

Still, it is premature to offer this as an explanation for the observed phase speeds before further fundamental studies of the string function are given. Aside from the obvious problem mentioned above (that the theory is for a two-layer ocean while the realistic ocean is continuously stratified) there are other problems. First, the string function calculated in this way is not the rest state on which the Rossby waves would propagate because the isopycnals are generally sloped. Hence, the string function is not the background string function 


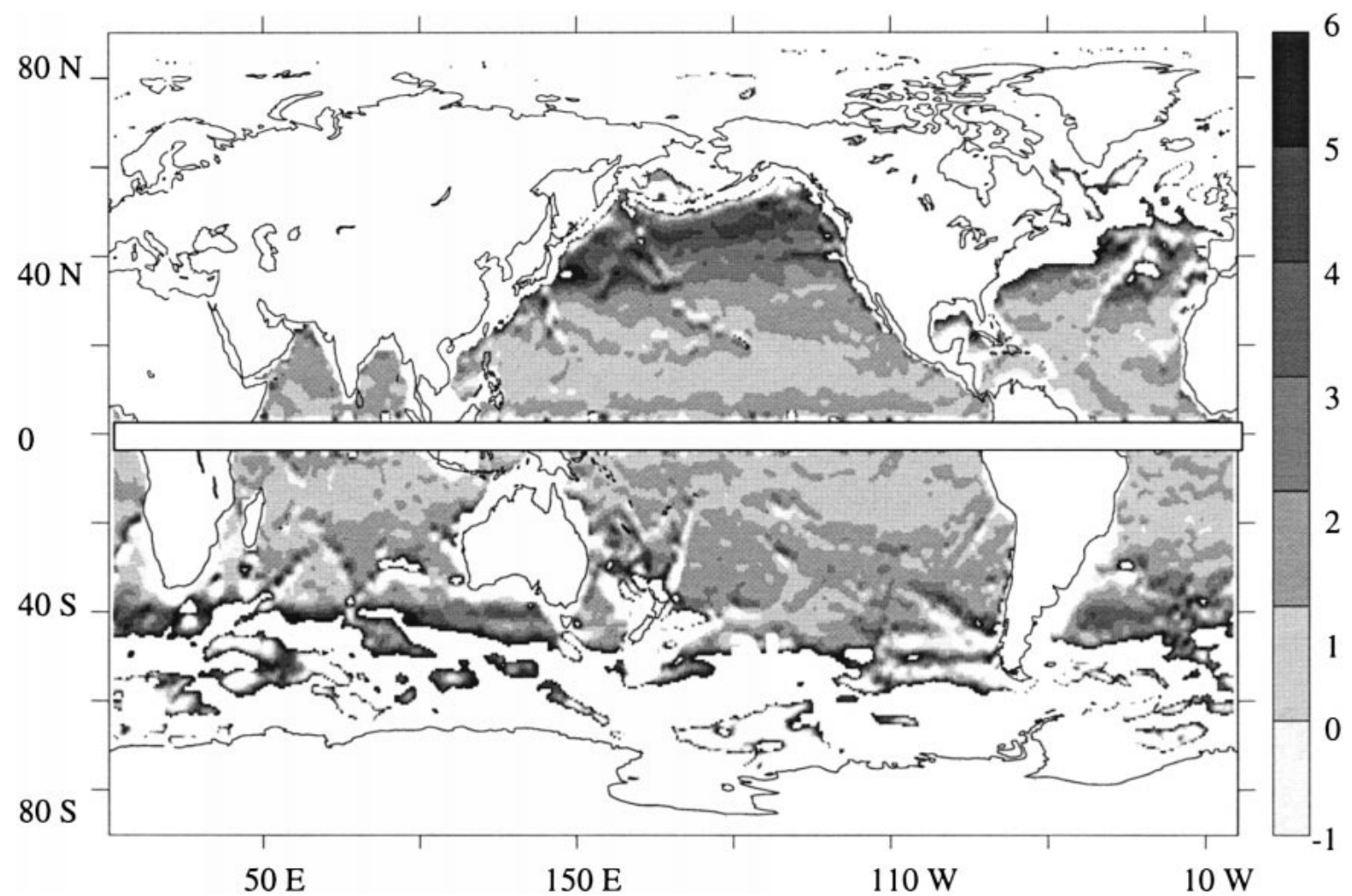

FIG. 3. Ratio of westward propagation speed expected from string function to that expected from classical theory [i.e., $\left.\partial_{y} \psi_{m} /\left(-\beta c^{2} / f^{2}\right)\right]$. The string function $\psi_{m}$ is shown in Fig. 2. Much of the Southern Ocean is off the high end of the grayscale.

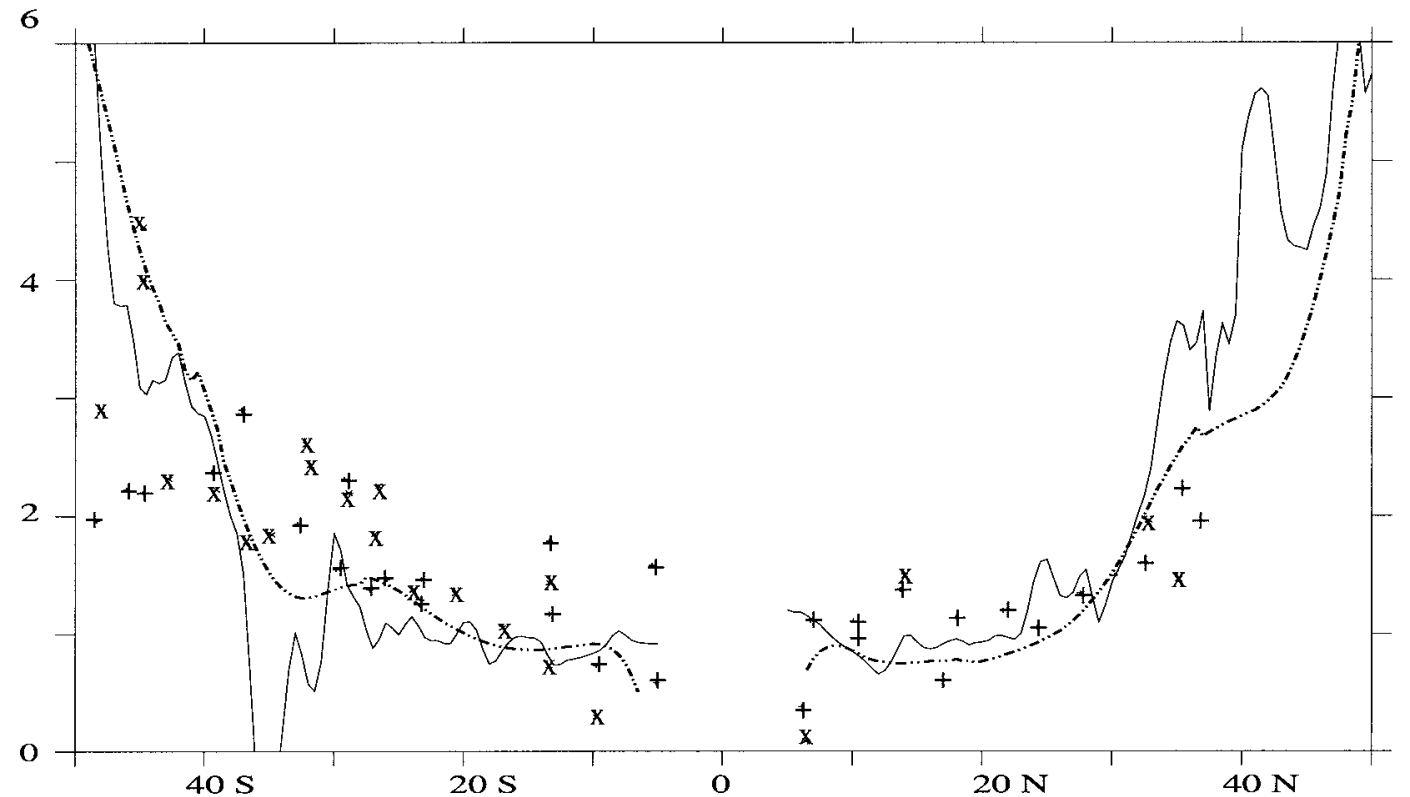

FIG. 4. Ratio of observed to theoretically expected westward phase speed of Rossby waves in the Atlantic and Indian Oceans (X) and Pacific (+) [redrawn from Chelton and Schlax (1996)]. Solid line shows the global zonal average of the westward phase speeds calculated from the string function as shown in Fig. 3 (dashed line is a Hanning low-pass of solid line). 
$\psi_{m_{o}}$ but rather the perturbed string function $\psi_{m}$ containing a part depending on $\tilde{m}$. In light of the process described in the text-related to the non-Doppler effect, whereby gradients of $\tilde{m}$ do not affect $\mathbf{c}_{m}$, this point must be examined more closely. Second, when topographic slope is a dominant factor, we saw in our simple estimate that the effect of the barotropic mode could be to increase the string velocity by up to a factor of 2 and this has not been considered here. The correlation seen in Fig. 4 and the simplicity with which the comparison could be made using the string function, do however provide a strong motivation for the further development of the string function.

Acknowledgments. This work was partially supported by the German Ministry of Education and Research $(\mathrm{BMBF})$ in the framework of the TIEFBIT Program.

\section{REFERENCES}

Chelton, D. B., and M. G. Schlax, 1996: Global observations of oceanic Rossby waves. Science, 272, 234-238.

—, R. A. DeSzoeke, M. G. Schlax, K. El Naggar, and N. Siwertz, 1998: Geographical variability of the first baroclinic rossby radius of deformation. J. Phys. Oceanogr., 28, 433-460.

Cornillon, P., R. Weyer, and G. Flierl, 1989: Translation velocity of warm core rings relative to the slope water. J. Phys. Oceanogr. 19, 1317-1332.

Cushman-Roisin, B., 1986: Frontal geostrophic dynamics. J. Phys. Oceanogr., 16, 132-143.

— E. E. Chassignet, and B. Tang, 1990: Westward motion of mesoscale eddies. J. Phys. Oceanogr., 20, 758-768.

Dewar, W. K., 1998: On "too-fast" baroclinic planetary waves in the general circulation. J. Phys. Oceanogr., 28, 1739-1758.

Flierl, G. R., 1977: The application of linear quasigeostrophic dynamics to Gulf Stream rings. J. Phys. Oceanogr., 7, 365-379.

_ 1984: Rossby wave radiation from a strongly nonlinear warm eddy. J. Phys. Oceanogr., 14, 47-58.

Kaese, R. H., and W. Zenk, 1996: Structure of the Mediterranean water and meddy characteristics in the northeastern Atlantic. The Warmwatersphere of the North Atlantic Ocean, Wolfgang Krauss, Ed., Gebrüder Borntraeger, 365-393.
Killworth, P. D., 1983: On the motion of isolated lenses on a betaplane. J. Phys. Oceanogr., 13, 368-376.

, D. B. Chelton, and R. A. De Szoeke, 1997: The speed of observed and theoretical long extratropical planetary waves. $J$. Phys. Oceanogr., 27, 1946-1966.

Matsuura, T., and T. Yamagata, 1982: On the evolution of nonlinear planetary eddies larger than the radius of deformation. J. Phys. Oceanogr., 12, 440-456.

McWilliams, J. C., and G. R. Flierl, 1979: On the evolution of isolated, nonlinear vortices. J. Phys. Oceanogr., 9, 1155-1182.

Mory, M., M. E. Stern, and R. W. Griffiths, 1987: Coherent baroclinic eddies on a sloping bottom. J. Fluid Mech., 183, 45-62.

Nof, D., 1981: On the $\beta$-induced movement of isolated baroclinic eddies. J. Phys. Oceanogr., 11, 1662-1672.

- 1983a: On the migration of isolated eddies with applications to Gulf Stream rings. J. Mar. Res., 41, 399-425.

- 1983b: The translation of isolated cold eddies on a sloping bottom. Deep-Sea Res., 30, 171-182.

Rhines, P. B., 1989: Deep planetary circulation and topography: Simple models of midocean flows. J. Phys. Oceanogr., 19, 14491470 .

, 1993: Oceanic general circulation: Wave and advection dynamics. Modelling Oceanic Climate Interactions, J. Willebrand and D. L. T. Anderson, Eds., Springer-Verlag, 67-150.

Shapiro, G. I., 1986: Dynamics of an isolated intrathermocline eddy (English translation). Oceanology, 26, 12-15.

Shi, C., and D. Nof, 1994: The destruction of lenses and generation of wodons. J. Phys. Oceanogr., 24, 1120-1136.

Smith, D. C., IV, and J. J. O'Brien, 1983: The interaction of a twolayer isolated mesoscale eddy with bottom topography. J. Phys. Oceanogr., 13, 1681-1697.

Straub, D. N., 1994: Dispersive effects of zonally varying topography on quasigeostrophic rossby waves. Geophys. Astrophys. Fluid Dyn., 75, 107-130.

Swaters, G. E., 1998: Numerical simulations of the baroclinic dynamics of density-driven coupled fronts and eddies on a sloping bottom. J. Geophys. Res., 103, 2945-2961.

— eddies on a sloping bottom. J. Fluid Mech., 223, 565-587.

Tyler, R. H., and R. Käse, 2000a: A “string function” for describing the propagation of large-scale energy anomalies in a rotating fluid. Geophys. Astrophys. Fluid Dyn., 92, 31-64.

, and R. Käse, 2000b: A numerical study of the string function using a primitive equation ocean model. Geophys. Astrophys. Fluid Dyn., 92, 65-83.

Whitehead, J. A., M. E. Stern, G. Flierl, and B. A. Klinger, 1990: Experimental observations of baroclinic eddies on a sloping bottom. J. Geophys. Res., 95, 9585-9610. 